\title{
Article
}

\section{Analysing agricultural diversification as a risk management strategy with the minimum regret model}

\author{
Jacob van Etten ${ }^{1}$ (D) \\ 1 Bioversity International (CGIAR); j.vanetten@cgiar.org \\ Version January 10, 2019 submitted to Preprints
}

\begin{abstract}
Diversification is an important strategy for managing risk in agricultural systems. Risk analysis can help to support farmers' diversification strategies, but existing analytical methods are complicated and little used. The minimum regret model helps to fill this gap. It provides a simple, transparent calculation procedure that can be executed with existing spreadsheet software. Regret is an important heuristic in the behavioural sciences and regret-based models are used in finance. The article presents the model with a numerical example. It also presents a framework to compare minimum regret portfolios with two limit cases (maximum utility and minimax regret). A case study illustrates the use of the model and the comparative framework.
\end{abstract}

Keywords: Agricultural diversification, risk management, regret, portfolio, scenario

\section{Introduction}

Agricultural diversification is widely practiced by farmers. Diversification can serve as a risk management strategy or "natural insurance", harnessing the buffering effects of different functional farm components. Farm components jointly reduce variation if they respond to time-variant biotic and abiotic stresses in different, complementary ways. Also, diversification can reduce market risk when different products have a low correlation between their respective market prices. Diversification can support climate adaptation, resilience and food security [1-3]. Examples of agricultural diversification are mixed crop-livestock systems [4], agroforestry [5], field scattering [6], varietal diversification [7,8], crop diversification [9], and intercropping [10].

Diversification for risk management is important enough to merit support from agricultural research and advisory services. Support can take the form of targeted introduction of genetic resources, discovering practices that use certain risk-reducing elements in farm portfolios, supporting policy design to encourage such practices, and opening new marketing channels for alternative products. Diversification does not automatically result in risk-reducing portfolios that address goals and concerns that farmers may have. Careful analysis is needed to ensure this.

Despite the importance of diversification for risk management, tools to analyze risk and compose risk-reducing portfolios are still seldom used in farm management or agricultural advisory services [11]. The literature explains the gap between theory and practice by blaming theory rather than practice: tools and analyses are generally too complicated, they focus on isolated aspects of farm risk management, lack contextual validity, make "heroic" assumptions about statistical distributions and uncertainty, produce results that do not match decision-making practice, or lack consideration of communication needs [12-14]. For practical use in agronomic design and analysis contexts, relatively simple tools are needed that facilitate quick assessment of different portfolios. Normally, design of agricultural systems does not achieve a single optimal solution but must consider trade-offs between multiple objectives [15]. Farmers will also consider the degree of protection against risk from other measures, including financial ones, and shift their farm 
portfolio accordingly [16]. Therefore, a quick calculation tool, in combination with other tools, would allow a systematic initial exploration of the solution space.

To address the need for a quick calculation tool, I present the minimum regret model $[17,18]$. Minimum regret is a computationally simple model to analyze and design portfolios, deciding about the combinations and proportions of the elements to include to reduce risk. The goal of this article is to introduce the minimum regret model to agricultural scientists. The focus is practical and the presentation is done through numerical examples. Mathematical details are presented in Appendix A. In the following section, I present the minimum regret model and discuss its theoretical background. I then probe how minimum regret can shed light on land use allocations in a case study on an agricultural system subjected to high climate risk. The case study illustrates the potential usefulness of the minimum regret model for portfolio analysis and design in agriculture.

\section{Minimum regret model}

The minimum regret model was first presented by Dembo and King $[17,18]$. The model builds on previous theory to construct portfolios, notably Modern Portfolio Theory [19]. It takes into account anticipated regret about opportunity loss [20-22]. The focus on regret provides protection against "downside risk", which is especially important when utility distributions are asymmetric [23].

The procedure behind the minimum regret model is intuitive. The decision-maker imagines plausible future scenarios. Then, for each of the scenarios, the decision-maker compares the utility of a given portfolio with the best possible choice. This gap in utility is called (opportunity) loss [20]. The decision-maker tries to minimize these loss values, giving more weight to the largest losses and to the scenarios that are more likely to occur. The combination of expected loss values translates into the decision-maker's anticipated regret. This procedure is cognitively not too taxing, as the decision-maker only compares with one imagined best portfolio under each scenario. This can be imagined as making comparisons with a neighbouring farmer who is clairvoyant, deciding a portfolio based on a perfect forecast. The scenarios are concrete combinations of conditions, which can be based on conditions in the recent past, but can also incorporate any other information, such as seasonal climate forecasts, price forecasts, and personal hunches about the future.

\section{Computational procedure}

The minimum regret model turns the intuitive procedure of comparing with a clairvoyant decision-maker into a sequence of computations. To calculate the value of regret, the model needs the following inputs:

- a payoff matrix that describes the payoff or return of different components when different scenarios ("states of nature") occur;

- the probabilities that each of the scenarios occurs;

- a portfolio, which is a series of values for relative shares for different portfolio components, which should sum to 1 .

With these inputs, the model returns the expectation of the regret that a decision-maker will experience in the future. To give more weight to larger losses, regret is a convex function of loss. I follow King [17] in using a quadratic function and calculate regret as the root mean square loss (see Appendix A for details). The model minimizes the expected regret by choosing optimal portfolio proportions for each component, which add up to 1 . A simple numerical example will help to explain the minimum regret model. Mathematical notation is given in the Appendix.

Table 1 gives a fictional payoff matrix of two different varieties that are available to a farmer, a modern variety (created by specialized plant breeders) and a farmer variety (selected and maintained by farmers). 

84 (Table 2).

Table 1. Example of a payoff matrix of two varieties for two scenarios and probability of occurrence

\begin{tabular}{lll}
\hline & Good year & Bad year \\
\hline Modern variety & $4 \mathrm{t} / \mathrm{ha}$ & $1 \mathrm{t} / \mathrm{ha}$ \\
Farmer variety & $2 \mathrm{t} / \mathrm{ha}$ & $2 \mathrm{t} / \mathrm{ha}$ \\
\hline Probability scenario occurs & 0.6 & 0.4 \\
\hline
\end{tabular}

As a first step, the payoff matrix is converted into a loss matrix by subtracting each value from the highest value in each column. The loss is the difference with the best possible option under each scenario

Table 2. Absolute loss matrix of two varieties for two scenarios and probability of occurrence.

\begin{tabular}{lcc}
\hline & Good year & Bad year \\
\hline Modern variety & $0 \mathrm{t} / \mathrm{ha}$ & $1 \mathrm{t} / \mathrm{ha}$ \\
$2 \mathrm{t} / \mathrm{ha}$ & $0 \mathrm{t} / \mathrm{ha}$ \\
\hline Farmer variety & 0.6 & 0.4 \\
\hline
\end{tabular}

85 From the loss matrix, the expected regret of any portfolio can be calculated. For example, the expected s6 regret of a simple 1:1 proportion between the two varieties (weights of 0.5 for each) would give the 87 calculation shown in Table 3.

Table 3. Absolute expected regret calculation for a portfolio of 50\% modern variety and 50\% farmer variety

\begin{tabular}{lll}
\hline & Good year & Bad year \\
\hline Modern variety (loss $\cdot$ share) & $0 \cdot 0.5=0$ & $1 \cdot 0.5=0.5$ \\
Farmer variety (loss $\cdot$ share) & $2 \cdot 0.5=1$ & $0 \cdot 0.5=0$ \\
Total loss (sum of above) & $0+1=1$ & $0.5+0=0.5$ \\
Absolute squared regret (squared total loss $\cdot$ probability) & $1^{2} \cdot 0.6=0.6$ & $0.5^{2} \cdot 0.4=0.1$ \\
\hline
\end{tabular}

Summing the quadratic regret values of Table 3 gives a value of 0.7 . To obtain a regret value on the original scale, the regret is expressed as the root mean square loss: $\sqrt{0.7}=0.84$ ton per hectare. The minimum regret can be calculated in the same way, changing the relative weight of the varieties in the portfolio to obtain the lowest expected regret value. In this case, it is easy to find an analytical solution. The sum of the weights should add up to 1 , so if $\mathrm{w}$ is the weight of the modern variety (a value between 0 and 1 ), then $1-w$ is the weight of the farmer variety. We can obtain the minimum regret by minimizing the following expression.

$$
\sqrt{0.6 \cdot((1-w) \cdot 2)^{2}+0.4 \cdot(w \cdot 1)^{2}}
$$

This expression has a minimum at $\mathrm{w}=0.86$. This means that the minimum regret portfolio has $86 \%$ of the modern variety and $14 \%$ of the farmer variety and an expected regret value of $0.59 \mathrm{t} / \mathrm{ha}$. In cases with 
97

more than two varieties, the equations become more complicated, but the optimization can be done using a generic optimizer, such as the Solver plug-in in Microsoft Excel.

Until now, I have only discussed absolute regret. The literature also describes relative regret, which is regret expressed as a ratio of the reference portfolio [16,22,24]. Both types of regret are potentially valid, depending on the context and goals of the decision-maker. Consider two years, one with a top yield of 2 $t /$ ha and a second year with a top yield of $4 t / h a$. An opportunity loss of $1 \mathrm{t} /$ ha would count equally for each year under absolute regret. Good years compensate for bad years. This is a plausible perspective when the farmer can deal with temporary losses using a loan, savings, stored food or income from other sources. Under relative regret, however, an opportunity loss of $1 \mathrm{t} /$ ha would be a much larger loss in the first year ( $50 \%$ of the reference) than in the second year ( $25 \%$ of the reference). A loss counts more heavily in a bad year than in a good year. Relative regret is applicable when farmers have limited access to resources and the farm is their main means of survival. For farmers in these circumstances, loss in a bad year is more likely to get the farm in the danger zone than a loss of the same size in a good year.

Given a loss matrix, the relative expected regret can be calculated in a similar way as demonstrated above for absolute regret. The result is shown in Table 4 . The only difference is that the total regret value of each year is divided by the square of the top yield for that same year. For this portfolio, the relative expected regret of the portfolio with equal proportions of the two varieties is $\sqrt{(0.0375+0.025)}=0.25$. This is to be interpreted as a proportion or percentage ("relative regret of $25 \%$ ").

Table 4. Relative expected regret calculation for a portfolio of 50\% modern variety and $50 \%$ farmer variety

\begin{tabular}{|c|c|c|}
\hline & Good year & Bad year \\
\hline Modern variety (loss · share) & $0 \cdot 0.5=0$ & $1 \cdot 0.5=0.5$ \\
\hline Farmer variety (loss $\cdot$ share) & $2 \cdot 0.5=1$ & $0 \cdot 0.5=0$ \\
\hline Total loss (sum of above) & $0+1=1$ & $0.5+0=0.5$ \\
\hline $\begin{array}{l}\text { Relative squared regret } \\
\text { (squared total loss / squared top yield) } \cdot \text { probability }\end{array}$ & $\left(1^{2} / 4^{2}\right) \cdot 0.6=0.0375$ & $\left(0.5^{2} / 2^{2}\right) \cdot 0.4=0.025$ \\
\hline
\end{tabular}

The variety portfolio can also be optimized for relative regret. The resulting portfolio has $60 \%$ of the high-yielding variety and $40 \%$ of the high-yielding variety and has a relative expected regret of $24 \%$. We can also calculate the absolute regret of this portfolio, which is $0.73 \mathrm{t} / \mathrm{ha}$. Minimizing the relative regret implies a sub-optimal absolute regret. The decision-maker must determine which type of regret corresponds to the context in which the portfolio should function.

\section{Comparative framework}

Real portfolios will deviate from ideal minimum-regret portfolios. In some cases, deviations will be due to external factors, including imperfect knowledge, technical constraints, or resource constraints. In other cases, however, deviations will may be inherent to decision-making and due to more relaxed or more conservative approaches to regret and downside risk. To cover these cases, I present two other types of models and their corresponding portfolios, which are limit cases of a more general model of regret. Together with the minimum regret model, they provide a mathematically coherent set of models (see Appendix A).

The first additional model is maximum expected utility, which does account for regret and just optimizes the long-term average outcome. This model corresponds to a decision-maker who is indifferent to risk or regret, focuses on long-term return and has capital or other income to survive bad years. The other model, minimax regret, represents the opposite extreme. Here the decision-maker focuses on the 
worst-case scenario and minimizes regret for this scenario. It represents a model with infinite regret, focusing exclusively on reducing the worst cases. It represents a farmer whose main worry is the survival of the farm, has no other ways to compensate losses and therefore avoids downside risk at all cost. Infinite regret does not consider the different probabilities of scenarios. This is appropriate under absolute uncertainty. Generally, farmers take into account the relative frequency of events for decision-making, so infinite regret can safely be considered a limit case. The role of the minimax model is therefore diagnostic or comparative but not prescriptive.

Minimum regret is in-between these two limit cases. This means that deviations towards weaker or stronger regret aversion can be interpreted in reference to these additional portfolios. Table 5 presents the resulting five scenarios and five metrics in an overview for the fictional case of the two-variety portfolio. Each portfolio optimizes one metric (shown in bold), but the same metric is also presented for the other portfolios. The expected utility of the regret-based portfolios is substantially below the optimum, showing that avoiding downside risk has a cost in the long term. However, even in the most extremely conservative approach to risk, the long-term utility would only be $0.4 \mathrm{t} /$ ha lower than the optimum of $2.8 \mathrm{t} / \mathrm{ha}$, a $14 \%$ reduction. Clearly, no metric would suggest that a farmer should ever plant less than $50 \%$ of land with the modern variety. It is evident that relative regret is a much more conservative criterion than absolute regret. In this example, minimum relative regret is even more conservative than minimax absolute regret. The potential reduction in expected absolute regret seems minimal, however, at most $0.04 \mathrm{t}$, but expected relative regret can be reduced by $25 \%$. Minimax relative regret is a very extreme position, as it recommends growing equal quantities of the two varieties, whereas intuitively it would make sense to grow some more of the modern variety, especially since good years are more frequent than bad years.

Table 5. Five diagnostic portfolios of two varieties with performance metrics. Values in bold are those optimized (minimized or maximized) by the respective model. Values for one hectare of land.

\begin{tabular}{lccccccc}
\hline Portfolio & $\begin{array}{c}\text { Proportion } \\
\text { modern } \\
\text { variety }\end{array}$ & $\begin{array}{c}\text { Proportion } \\
\text { farmer } \\
\text { variety }\end{array}$ & $\begin{array}{c}\text { Expected } \\
\text { utility (t) }\end{array}$ & $\begin{array}{c}\text { Expected } \\
\text { absolute } \\
\text { regret (t) }\end{array}$ & $\begin{array}{c}\text { Expected } \\
\text { relative } \\
\text { regret }\end{array}$ & $\begin{array}{c}\text { Worst } \\
\text { absolute } \\
\text { regret (t) }\end{array}$ & $\begin{array}{c}\text { Worst } \\
\text { relative } \\
\text { regret }\end{array}$ \\
\hline $\begin{array}{l}\text { Maximum } \\
\text { expected utility }\end{array}$ & 1.00 & 0.00 & $\mathbf{2 . 8 0}$ & 0.63 & 0.32 & 1.00 & 0.50 \\
$\begin{array}{l}\text { Minimum } \\
\text { absolute regret }\end{array}$ & 0.86 & 0.14 & 2.69 & $\mathbf{0 . 5 9}$ & 0.28 & 0.86 & 0.43 \\
$\begin{array}{l}\text { Minimum } \\
\text { relative regret }\end{array}$ & 0.60 & 0.40 & 2.48 & 0.73 & $\mathbf{0 . 2 4}$ & 0.80 & 0.30 \\
$\begin{array}{l}\text { Minimax } \\
\text { absolute regret }\end{array}$ & 0.67 & 0.33 & 2.53 & 0.67 & 0.25 & 0.67 & 0.33 \\
$\begin{array}{l}\text { Minimax } \\
\text { relative regret }\end{array}$ & 0.50 & 0.50 & 2.40 & 0.84 & 0.25 & 1.00 & $\mathbf{0 . 2 5}$ \\
\hline
\end{tabular}

\section{Potential contribution of the minimum regret model}

Minimum regret is an attractive model for several reasons. The first reason is that it has support in theory. Anticipated regret is an important heuristic that explains experimental results from decision-making experiments [21,22]. Such heuristics have become more central in recent research on human decision-making, which studies heuristics as cost-effective shortcuts to reach rational decisions $[25,26]$. Minimizing expected regret is a rational way to think about risk and has an important role in financial risk management $[27,28]$. It has been shown to be equivalent to Conditional Value-at-Risk, a 
"coherent" state-of-the-art risk metric in finance [29,30], which has already been applied in agriculture to generate variety portfolio recommendations [31].

Minimum regret is also attractive because of its simple calculation procedure. The scenario-based focus of the minimum regret model as presented here stays close to the input data [32]. It does not reduce the uncertainty in the input data, but it makes the modelling process highly transparent (visible on a spreadsheet), so that the limitations are relatively easy to understand. In many cases, a simple model with known limitations is a better starting point than a more comprehensive model that is more difficult to understand and contextualize. Applications of insights from portfolio construction exercises are unlikely to be straightforward, as decisions will often be constrained by farm-specific factors or informed by local knowledge and innovative solutions that are difficult to foresee [33,34]. A simple calculation tool should help decision-makers to move between analysis and synthesis quickly, focusing on risk as one of several aspects. For risk analysis, the minimum regret model would serve as a quick calculation tool. For other aspects, decision-makers could use similar calculation tools, rules of thumb, and qualitative assessments, especially in the first steps of the design process. DeKay and Brown [35] argue that in bioclimatic architecture $80 \%$ of the energy savings are made in the first sketch, which therefore needs to be supported by broad-ranging, quick analyses. Similarly, in agricultural systems design, simple calculation tools serve to explore a broad range of options that would be difficult to assess with more demanding modelling tools. Another advantage of analytical simplicity is that the results are easy to communicate.

Despite this aim for initial analytical simplicity, there is nothing holding back the analyst from extending the minimum regret model with more sophisticated methods. For example, bootstrapping can serve to assess uncertainty in estimated parameters and portfolios, as is done with comparable data-driven methods [11]. Therefore, the minimum regret model could be gradually extended and provide a bridge between practical agronomic decision-making and more sophisticated modelling exercises.

\section{Case study: Crop portfolio for inter-annual rainfall variation}

As a first illustration of the minimum regret model and the comparative framework, I focus on an agricultural system in which climate risk is a main factor for farmers' crop portfolio decisions. The case study is based on information provided by Matsuda [36], who did a detailed quantitative study of a farming system in the central part of Myanmar. In this area, farming households grow a diverse portfolio of cash crops and use the income to buy rice grown elsewhere. Agricultural diversification characterizes these highly commercial farming systems. The analysis will focus on how farmers construct farm portfolios of three main cropping systems: (1) cotton-pigeonpea, (2) sesame, and (3) legumes (excluding pigeonpea). There was generally no double cropping in the area, so each year land is assigned to one of these cropping systems.

Matsuda provides data on crop allocations, yields and market prices for 7 years (2002-2008) [36]. My analysis uses these past years as equiprobable scenarios that inform farmers' portfolios. In other words, I assume that farmers create portfolios to face a year that is randomly drawn from the previous 7 years. To obtain a consistent payoff table, I calculated revenue per unit of land for the three cropping systems and corrected revenues for inflation (Appendix B). Variations in revenue reflect the combination of variation in both yield and market prices, but yield variation dominates due to the high seasonal variability in rainfall. Table 7 shows the revenue data. Initially, the analysis makes the simplifying assumption that farmers allocate land to different cropping systems considering only crop revenue and its variation. The calculations can be reproduced with the Excel spreadsheets provided as Supplementary Information. 
Table 6. Revenue for three cropping systems in Central Myanmar, derived from data in [36]. In euro per hectare (2009 inflation-corrected prices).

\begin{tabular}{rrrr}
\hline Year & $\begin{array}{c}\text { Cotton- } \\
\text { pigeonpea }\end{array}$ & Sesame & $\begin{array}{r}\text { Legumes } \\
\text { (except } \\
\text { pigeonpea) }\end{array}$ \\
\hline 2002 & 30 & 154 & 40 \\
2003 & 218 & 207 & 97 \\
2004 & 230 & 93 & 184 \\
2005 & 151 & 117 & 109 \\
2006 & 289 & 48 & 244 \\
2007 & 420 & 217 & 248 \\
2008 & 171 & 204 & 150 \\
\hline
\end{tabular}

From the payoff data in Table 6, I generated five diagnostic portfolios, shown in Table 7. Table 7 compares these theoretical predictions with the empirical data ("Average observed portfolio" in the bottom row). The diagnostic portfolios all correctly predict that cotton-pigeonpea is the most important cropping system. The regret-based portfolios correctly assign a non-zero share to sesame, which is omitted completely in the maximum utility model. None of the portfolios, however, includes a substantial share for legumes. Only the minimax absolute regret model gives it a small share, yet much lower than observed. It is easy to trace back this pattern to the payoff table. In none of the years, legumes outperform both cotton-pigeonpea and sesame at the same time. A combination of cotton-pigeonpea and sesame protects well against risk.

Table 7. Five diagnostic portfolios of two varieties with performance metrics. Values in bold are those optimized (minimized or maximized) by the respective model. Values for one hectare of land.

\begin{tabular}{lcccccccc}
\hline Portfolio & $\begin{array}{c}\text { Proportion } \\
\text { cotton- } \\
\text { pigeonpea }\end{array}$ & $\begin{array}{c}\text { Proportion } \\
\text { sesame }\end{array}$ & $\begin{array}{c}\text { Proportion } \\
\text { legumes } \\
\text { (except } \\
\text { pigeonpea) }\end{array}$ & $\begin{array}{c}\text { Expected } \\
\text { utility (t) }\end{array}$ & $\begin{array}{c}\text { Expected } \\
\text { absolute } \\
\text { regret }\end{array}$ & $\begin{array}{c}\text { Expected } \\
\text { relative } \\
\text { regret }\end{array}$ & $\begin{array}{c}\text { Worst } \\
\text { absolute } \\
\text { regret (t) }\end{array}$ & $\begin{array}{c}\text { Worst } \\
\text { relative } \\
\text { regret }\end{array}$ \\
\hline $\begin{array}{l}\text { Maximum } \\
\text { expected utility }\end{array}$ & 1.00 & 0.00 & 0.00 & 216 & 49 & 0.31 & 124 & 0.81 \\
$\begin{array}{l}\text { Minimum } \\
\text { absolute regret }\end{array}$ & 0.88 & 0.12 & 0.00 & 208 & 46 & 0.28 & 109 & 0.71 \\
$\begin{array}{l}\text { Minimum } \\
\text { relative regret }\end{array}$ & 0.66 & 0.34 & 0.00 & 193 & 55 & $\mathbf{0 . 2 5}$ & 82 & 0.53 \\
$\begin{array}{l}\text { Minimax } \\
\text { absolute regret }\end{array}$ & 0.60 & 0.33 & 0.07 & 189 & 59 & 0.26 & 83 & 0.54 \\
$\begin{array}{l}\text { Minimax } \\
\text { relative regret }\end{array}$ & 0.51 & 0.49 & 0.00 & 183 & 74 & 0.27 & 118 & $\mathbf{0 . 4 1}$ \\
$\begin{array}{l}\text { Average } \\
\text { bbserved portfolio }\end{array}$ & 0.51 & 0.25 & 0.24 & 184 & 61 & 0.28 & 92 & 0.60 \\
\hline
\end{tabular}

The underprediction of the share of legumes probably implies that these crops are not included in the system to reduce the risk of revenue per hectare. A possible reason for the prominence of legumes in the portfolio is that they help to distribute labour demand more evenly across the year and/or help to reduce overall labour demand. Legumes have a short production cycle and can be sown at different times, avoiding the harvest period of the other crops, as shown by the cropping calendar presented by Matsuda [36]. 
Assuming that legumes are included in the portfolio for reasons unrelated to revenue risk management, I fixed the proportion of legumes at the observed value of $24 \%$ and then reran the optimizations (Table 8). With this constraint added, the minimum absolute regret model predicts that $74 \%$ land will be planted to cotton-pigeonpea and $2 \%$ will be under sesame, far from the observed values. For the minimum relative regret model, this is $49 \%$ for cotton-pigeonpea and $27 \%$ for sesame, close to reality. This suggests minimum relative regret best reflects farmers' choices. Relative regret is reasonable in this context, as the possibility from inter-annual risk transfer is limited. Compared to maximum utility, the observed portfolio achieves a reduction in both expected and worst relative regret, but not in expected absolute regret (Table 7).

Table 8. Constrained minimum regret portfolios, following the same procedure as in Table 7 , but setting the proportion of legumes to 0.24 .

\begin{tabular}{lcccccccc}
\hline Portfolio & $\begin{array}{c}\text { Proportion } \\
\text { cotton- } \\
\text { pigeonpea }\end{array}$ & $\begin{array}{c}\text { Proportion } \\
\text { sesame }\end{array}$ & $\begin{array}{c}\text { Proportion } \\
\text { legumes } \\
\text { (except } \\
\text { pigeonpea) }\end{array}$ & $\begin{array}{c}\text { Expected } \\
\text { utility (t) }\end{array}$ & $\begin{array}{c}\text { Expected } \\
\text { absolute } \\
\text { regret }\end{array}$ & $\begin{array}{c}\text { Expected } \\
\text { relative } \\
\text { regret }\end{array}$ & $\begin{array}{c}\text { Worst } \\
\text { absolute } \\
\text { regret (t) }\end{array}$ & $\begin{array}{c}\text { Worst } \\
\text { relative } \\
\text { regret }\end{array}$ \\
\hline $\begin{array}{l}\text { Minimum } \\
\text { absolute regret }\end{array}$ & 0.74 & 0.02 & $0.24^{*}$ & 199 & 52 & 0.31 & 119 & 0.77 \\
$\begin{array}{l}\text { Minimum } \\
\text { relative regret }\end{array}$ & 0.49 & 0.27 & $0.24^{*}$ & 182 & 96 & 0.28 & 96 & 0.57 \\
\hline *Fixed, non-optimized value. & & & & & & & &
\end{tabular}

26

$$
\text { be }
$$$$
\text { pri }
$$$$
\text { anc }
$$
and costs throughout the year and land suitability. Similar elicitation exercises can also explore the effects of other risk management measures such as climate forecasts, crop insurance, crop storage, stress-tolerant varieties, and new crops. These new measures can be evaluated by repetitively changing the payoff matrix and rerunning the optimization.

\section{Discussion}

The case study shows how the minimum regret model can serve as a relatively simple calculation tool for an exploratory analysis of diversified portfolios. Data for a limited period of seven years was available. A more definitive analysis would need a comprehensive set of scenarios and calibrated probabilities. More data would also be needed to provide evidence on the relative adequacy of minimum regret versus other theories of risk in this case. Such data are rarely at hand for practical decision-making situations, however. The case study example shows that even without precise calibration or a comprehensive representation of the farming system, insights on the crop portfolio can be extracted from the data. The comparative framework confirmed that the observed deviations are not due to different degrees of regret aversion, but to other reasons. On the basis of this, specific hypotheses can be formulated that can guide next steps in an iterative exploration. The case study illustrates how minimum regret can be a useful addition to the toolbox of agricultural systems analysts. As analysts gain more experience with the model in practical contexts, its usefulness should become clearer, specifically in relation to the practical reasoning of farmers to analyse risks and select diversified portfolios. 


\section{Materials and Methods}

All data for the case study were derived from [36]. In the study area, farmers mainly grow the following crops: pigeonpea, cotton, sesame, and several minor pulses, including suntani/suntapya/butter beans (Phaseolus lunata), green gram (Vigna radiata), chickpea (Cicer arietinum), and lablab (Lablab purpurea). Farmers generally intercrop cotton and pigeonpea, so data for these two crops were combined to analyse it as a single cropping system. The minor pulses were also considered as one cropping system, following [36].

Inflation influenced market prices strongly in this period, precluding a direct comparison between years. Rice prices increased more than fourfold during this period. Since buying rice is a main livelihood goal for farmers in the area, I used the rice price reported by [36] as an index to standardize the revenue data. I divided the revenue by the rice price of the corresponding year, which expressed the revenue in units of rice. I then multiplied this with the rice price of 2009 and converted to euros per hectare. Two data points for the price of legumes were missing. I imputed these values with zero-intersect linear regression, using the strong correlation between legumes and pigeonpea market prices in the other years $(\mathrm{r}=0.99)$. I combined price data from each year with the yield data from the previous year. The calculation can be traced in the Supplementary Information (Excel file).

Funding: This research was undertaken as part of the CGIAR Research Program on Roots, Tubers and Bananas (RTB).

Acknowledgments: I thank Dr M. Matsuda for his generous explanations that improved my understanding of the case study. Any remaining errors are my own.

Conflicts of Interest: The author declares no conflict of interest. The funders had no role in the design of the study; in the collection, analyses, or interpretation of data; in the writing of the manuscript, or in the decision to publish the results.

\section{Appendix}

Minimum regret is calculated following equations A1 (absolute regret) and A2 (relative regret).

$$
\begin{gathered}
\underset{w_{i}}{\arg \min } E\left(R_{a b s}\right)=\sum_{s \in S} p_{s} \cdot\left(\sum_{i \in N} w_{i} \cdot\left(\max \left\{r_{s, 1}, r_{s, 2}, r_{s, 3}, \ldots, r_{s, n}\right\}-r_{s, i}\right)\right)^{2} \\
\underset{w_{i}}{\arg \min } E\left(R_{r e l}\right)=\sum_{s \in S} p_{s} \cdot \frac{\left(\sum_{i \in N} w_{i} \cdot\left(\max \left\{r_{s, 1}, r_{s, 2}, r_{s, 3}, \ldots, r_{s, n}\right\}-r_{s, i}\right)\right)^{2}}{\max \left\{r_{s, 1}, r_{s, 2}, r_{s, 3}, \ldots, r_{s, n}\right\}^{2}} \\
\text { (A1) and (A2) subject to } \quad \sum_{i \in N} w_{i}=1 \quad w_{i} \geq 0 \quad \sum_{s \in S} p_{s}=1
\end{gathered}
$$

$E\left(R_{a b s}\right)$ is the expected (quadratic) absolute regret.

$E\left(R_{r e l}\right)$ is the expected (quadratic) relative regret.

$S$ is a scenario from the set of scenarios $S$.

Set $S$ contains all possible future scenarios.

$i$ is one of the $n$ portfolio elements in set $N$.

$w_{i}$ is the weight of the $i$ th element.

$r_{s, i}$ is the return (yield, revenue, profit) of element $i$ under scenario $s$.

$p_{s}$ is the probability that scenario $s$ occurs.

For practical use of regret-based portfolios, I focus on this version, which uses a quadratic transform of loss $[17,18,37]$. I report regret as the square mean root expected regret: $\sqrt{\left(E\left(R_{a b s}\right)\right)}$ and $\sqrt{\left(E\left(R_{r e l}\right)\right)}$. The use of quadratic loss functions has been criticized as convexity is not always desirable (having larger losses count more heavily) ([38], Section 2.4.2.1). In this context, however, the convexity of the loss 
function is inherent to the focus on regret. A linear loss function corresponds to the expected utility. A quadratic function is an obvious choice, because it has a close conceptual link with the mean-variance model in Modern Portfolio Theory [17]. Geometrically, the root mean square loss can be understood as the downward Euclidean distance from a "clairvoyant" portfolio (in analogy to the standard deviation as the Euclidean distance of a sample from the mean). The quadratic loss function should be easy to understand for those who are familiar with the root mean square error (RMSE). Expressing regret as the root mean square loss transforms it back to the same scale as the variable in the payoff matrix in the case of absolute values (revenue, yield per hectare, etc.).

The minimum regret model can be extended with several parameters. Hayashi [39] proposes a "smooth" model that leaves the exponent as a free parameter $(\alpha)$ Equation A3 shows this model, following the same notation as for equations A1 and A2.

$$
\begin{aligned}
\underset{w_{i}}{\arg \min } E\left(R_{a b s, \alpha}\right)= & \sum_{s \in S} \sum_{i \in N} p_{s} \cdot\left(w_{i} \cdot\left(\max \left\{r_{s, 1}, r_{s, 2}, r_{s, 3}, \ldots, r_{s, n}\right\}-r_{s, i}\right)\right)^{\alpha} \\
\text { subject to } & \sum_{i \in N} w_{i}=1 \quad w_{i} \geq 0 \quad \sum_{s \in S} p_{s}=1 \quad \alpha \geq 1
\end{aligned}
$$

So instead of having $\alpha=2$ to obtain King's [17] version of minimum regret, Hayashi's [39] model changes the value of $\alpha$ according to the preference of the decision-maker. Presenting a series of portfolios based on different intermediate parameter values, however, has limited value in facilitating decision-making. It is difficult for a decision-maker to define a priori an optimal or desirable level of regret aversion, or to attach a common-sense meaning to an intermediate value of a rather abstract parameter. Instead, I focus on two limit cases. One limit case is obtained when the parameter $\alpha$ in Hayashi's [39] model is set to 1 . This results in linear loss minimization, which is equivalent to the maximum expected utility model. In this model, regret does not play a role. The other limit case would be to let parameter $\alpha$ approach infinity $(\infty)$. This is equivalent to minimax regret, which focuses on limiting regret in the worst-case scenario [20,40]. Minimax regret does not consider the probabilities of the different scenarios, since it gives only weight to the worst-case scenario. For both models, an absolute and a relative loss function can be used. Together, these different values of $\alpha$ produce a set of mathematically coherent, discrete portfolios. The following formulas correspond to maximum utility (A4), minimax absolute regret (A5) and minimax relative regret (A6).

$$
\begin{gathered}
\underset{w_{i}}{\arg \max } E(U)=\sum_{s \in S} \sum_{i \in N} p_{s} \cdot w_{i} \cdot r_{s, i} \\
\underset{w_{i}}{\arg \min } E\left(R_{a b s, \max }\right)=\max _{s \in S} \sum_{i \in N} w_{i} \cdot\left(\max \left\{r_{s, 1}, r_{s, 2}, r_{s, 3}, \ldots, r_{s, n}\right\}-r_{s, i}\right) \\
\underset{w_{i}}{\arg \min } E\left(R_{\text {rel.max }}\right)=\max _{s \in S} \sum_{i \in N} w_{i} \cdot \frac{\max \left\{s, 1, r_{s, 2}, r_{s, 3}, \ldots, r_{s, n}\right\}-r_{s, i}}{\max \left\{r_{s, 1}, r_{s, 2}, r_{s, 3}, \ldots, r_{s, n}\right\}} \\
(\mathrm{A} 4) \text { - (A6) subject to } \quad \sum_{i \in N} w_{i}=1 \quad w_{i} \geq 0 \quad \sum_{s \in S} p_{s}=1
\end{gathered}
$$

1. Kremen, C.; Iles, A.; Bacon, C. Diversified farming systems: an agroecological, systems-based alternative to modern industrial agriculture. Ecology and Society 2012, 17, 44. 


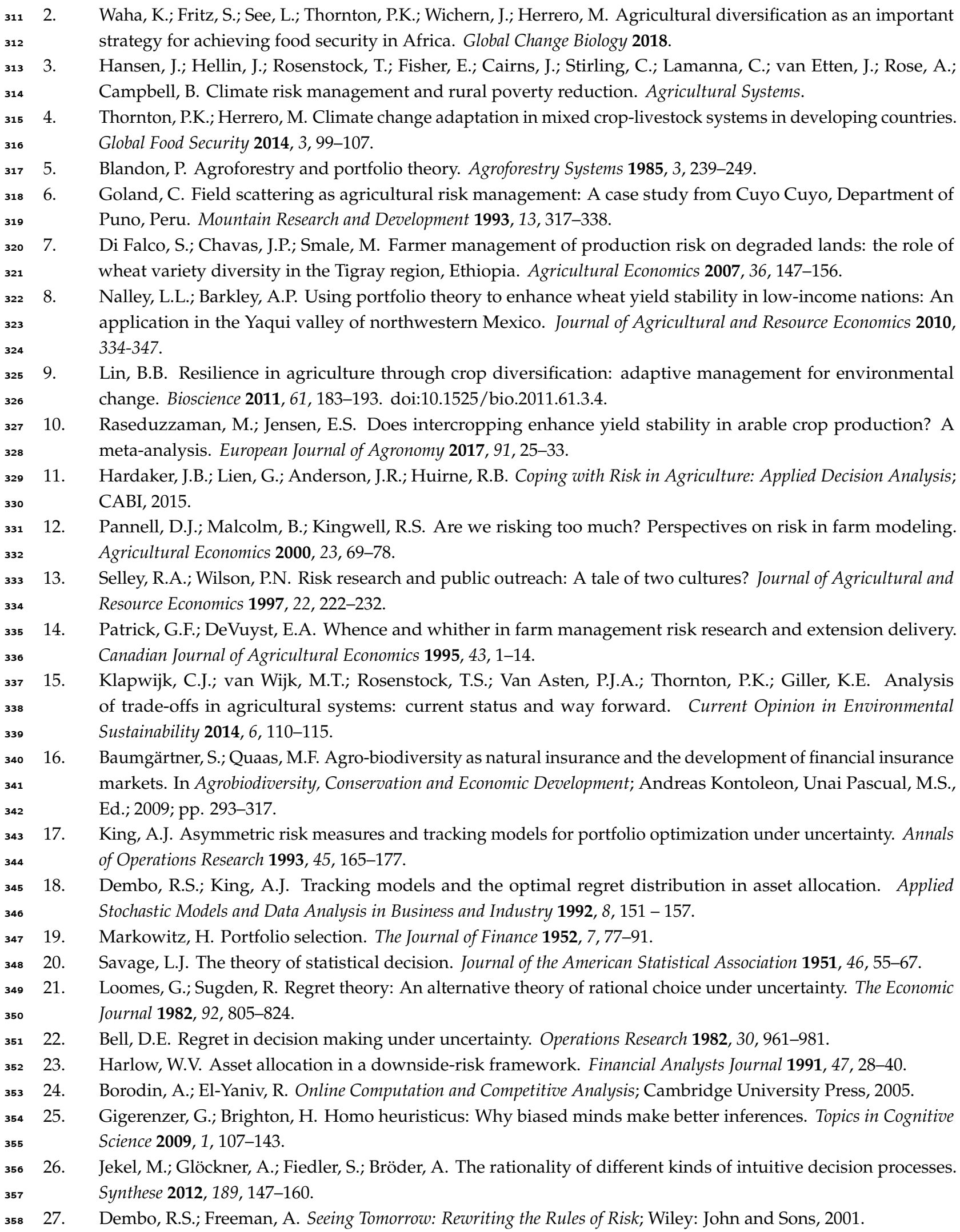


28. Michenaud, S.; Solnik, B. Applying regret theory to investment choices: Currency hedging decisions. Journal of International Money and Finance 2008, 27, 677-694.

29. Testuri, C.E.; Uryasev, S. On relation between expected regret and Conditional Value-at-Risk. In Handbook of Computational and Numerical Methods in Finance; Rachev, S.T., Ed.; Springer: Boston, MA, 2004; pp. 361-372.

30. Szegö, G. Measures of risk. European Journal of Operational Research 2005, 163, 5-19.

31. Sukcharoen, K.; Leatham, D. Mean-Variance Versus Mean-Expected Shortfall Models: An Application To Wheat Variety Selection. Journal of Agricultural and Applied Economics 2016, 48, 148-172.

32. Dembo, R.S. Scenario optimization. Annals of Operations Research 1991, 30, 63-80.

33. Suppe, F. The limited applicability of agricultural research. Agriculture and Human Values 1987, 4, 4-14.

34. Fujisaka, S. Research: help or hindrance to good farmers in high risk systems? Agricultural Systems 1997, $54,137-152$.

35. DeKay, M.; Brown, G.Z. Sun, Wind, and Light: Architectural Design Strategies; Wiley: John \& Sons, 2013.

36. Matsuda, M. Upland farming systems coping with uncertain rainfall in the central dry zone of Myanmar: how stable is indigenous multiple cropping under semi-arid conditions? Human Ecology 2013, 41, 927-936. doi:http://dx.doi.org/10.1007/s10745-013-9604-x.

7. Hennig, C.; Kutlukaya, M. Some thoughts about the design of loss functions. REVSTAT-Statistical Journal 2007, $5,19-39$.

Berger, J.O. Statistical Decision Theory and Bayesian Analysis; Springer: Science \& Business Media, 2013.

39. Hayashi, T. Regret aversion and opportunity dependence. Journal of Economic Theory 2008, 139, 242-268.

40. Hayashi, T. Context dependence and consistency in dynamic choice under uncertainty: the case of anticipated regret. Theory and Decision 2011, 70, 399-430. 